\title{
Erratum to: An offer you can't refuse: consumer perceptions of sales pressure
}

\author{
James J. Zboja ${ }^{1}$ - Ronald A. Clark ${ }^{2}$ - Diana L. Haytko ${ }^{3}$
}

Published online: 30 April 2016

(C) Academy of Marketing Science 2016

Erratum to: J. of the Acad. Mark. Sci.

DOI 10.1007/s11747-015-0468-Z

Tables 3 and 4 in the original version of this article contained

mistakes in the entries. The correct tables are shown below:

Table 3 Means, standard deviations, correlations, reliabilities, and convergent/discriminant validity assessment of the measurement model

\begin{tabular}{|c|c|c|c|c|c|c|c|c|c|c|}
\hline Variable & $M$ & $S D$ & $A V E$ & 1 & 2 & 3 & 4 & 5 & 6 & 7 \\
\hline 1. POSP (A) & 2.47 & 1.30 & 0.60 & .86 & 0.55 & 0.05 & 0.15 & 0.04 & 0.36 & 0.28 \\
\hline 2. POSP (D) & 2.51 & 1.45 & 0.75 & $0.74 * *$ & .90 & 0.04 & 0.17 & 0.01 & 0.29 & 0.21 \\
\hline 3. SKNOW & 5.44 & 1.51 & 0.88 & $-0.22 * *$ & $-0.19 * *$ & .98 & 0.03 & 0.00 & 0.26 & 0.24 \\
\hline 4. TIME & 3.44 & 1.63 & 0.66 & $0.39 * *$ & $0.41 * *$ & $-0.17 * *$ & .85 & 0.02 & 0.15 & 0.15 \\
\hline 5. SCPK & 5.93 & 0.66 & 0.63 & $-0.19 * *$ & $-0.12 *$ & 0.06 & $-0.13^{*}$ & .83 & 0.02 & 0.02 \\
\hline 6. SAT & 5.09 & 1.41 & 0.77 & $-0.60 * *$ & $-0.54 * *$ & $0.51 * *$ & $-0.39 * *$ & $0.13^{*}$ & .94 & 0.62 \\
\hline 7. TRUST & 4.89 & 1.26 & 0.75 & $-0.53 * *$ & $-0.46^{* *}$ & $0.49 * *$ & $-0.39 * *$ & $0.13^{*}$ & $0.79 * *$ & .90 \\
\hline
\end{tabular}

POSP $(A, D)$ Perceptions of Sales Pressure (Aggressive, Directive), SKNOW Salesperson Product Knowledge, TIME Time Pressure, SCPK SelfConfidence, Persuasion Knowledge, SAT Satisfaction with Salesperson, TRUST Trust in Salesperson

- Listwise $n=275, A V E$ Average variance extracted

- Construct reliabilities are provided on the diagonal in bold

- Shared variances are reported above the diagonal

- Pearson correlations are below the diagonal

** Correlation is significant at the 0.01 level (2-tailed)

* Correlation is significant at the 0.05 level (2-tailed)

The online version of the original article can be found at http://dx.doi.org/ 10.1007/s11747-015-0468-z.

Diana L. Haytko

dhaytko@fgcu.edu

1 Department of Management \& Marketing, Collins College of Business, University of Tulsa, Tulsa, OK 74104-9700, USA

2 Department of Marketing, College of Business, Missouri State University, Springfield, MO 65897, USA

3 Department of Marketing, Lutgert College of Business, Florida Gulf Goast University, Fort Myers, FL 33965, USA 
Table 4 Structural model results maximum likelihood estimates

\begin{tabular}{llll}
\hline Hypothesized Path & Path Coefficient & $R^{2}$ & Support \\
\hline 1a. Salesperson Knowledge $\rightarrow$ POSP (A) & $-0.14^{*}$ & 0.31 & Supported \\
1b. Salesperson Knowledge $\rightarrow$ POSP (D) & -0.11 & 0.29 & Not Supported \\
2a. Time Pressure $\rightarrow$ POSP (A) & $0.47^{*}$ & & Supported \\
2b. Time Pressure $\rightarrow$ POSP (D) & $0.49^{*}$ & & Supported \\
3a. Self-Confidence (PK) $\rightarrow$ POSP (A) & $-0.14^{*}$ & & Supported \\
3b. Self-Confidence (PK) $\rightarrow$ POSP (D) & -0.07 & Not Supported \\
4a. Salesperson Knowledge $\rightarrow$ Satisfaction & $0.38^{*}$ & & Supported \\
4b. Time Pressure $\rightarrow$ Satisfaction & $-0.14^{*}$ & & Supported \\
4c. POSP (A) $\rightarrow$ Satisfaction & $-0.52^{*}$ & Supported \\
4d. POSP (D) $\rightarrow$ Satisfaction & -0.03 & Not Supported \\
5a. Salesperson Knowledge $\rightarrow$ Trust & $0.38^{*}$ & Supported \\
5b. Time Pressure $\rightarrow$ Trust & $-0.17^{*}$ & Supported \\
5c. POSP $(\mathrm{A}) \rightarrow$ Trust & $-0.50^{*}$ & & Supported \\
5d. POSP $(\mathrm{D}) \rightarrow$ Trust & 0.02 & Not Supported \\
Structural Model Results: & & & \\
$X^{2}(d f=258, n=275)=675.49, \mathrm{p}<.05$ & & & \\
CFI $=.97 ;$ TLI $=.97 ;$ IFI $=.97 ;$ RMSEA $=.077$ & & & \\
\hline
\end{tabular}

